\title{
An energy-efficient multistage charge pump for MEMS microphone
}

\author{
Rongshan Weia) and Jue Wang \\ College of Physics and Information Engineering, Fuzhou University, \\ Fuzhou, Fujian, 350116, China
}

a)wrs08@fzu.edu.cn

\begin{abstract}
This paper proposes an energy-efficient, 12-stage charge pump with constant threshold voltage. This new charge pump utilizes 2 pairs of non-overlapping clocks. Under control of an extra pair of clocks, each charge pump cell (CPC) is equivalent to a switch. Without reduction by a threshold voltage and the limitation of body effects, a higher output voltage than that of the traditional charge pump is obtained by the proposed circuit, which is fabricated in a $0.18 \mu \mathrm{m}$ complementary metal-oxide semiconductor (CMOS) process. With an input voltage from $0.7 \mathrm{~V}$ to $1.4 \mathrm{~V}$, the output voltage range is from $5.8 \mathrm{~V}$ to $12.5 \mathrm{~V}$. With a $2-\mathrm{pF}$ load capacitor, the power consumption is only $62.7 \mu \mathrm{W}$ at maximum DC output voltage of $12.5 \mathrm{~V}$. This circuit is intended to generate an on-chip bias voltage for a microelectromechanical systems (MEMS) microphone.
\end{abstract}

Keywords: charge pump, MEMS microphone, body effect, threshold voltage

Classification: Electron devices, circuits and modules

\section{References}

[1] A. Cheng: Design of a Readout Scheme for a MEMS Microphone (Master of Science, Delft University of Technology, Delft, 2009).

[2] J. Weigold, et al.: "A MEMS condenser microphone for consumer applications," MEMS (2006) 86 (DOI: 10.1109/MEMSYS.2006.1627742).

[3] S.-S. Je, et al.: "A directional capacitive MEMS microphone using nanoelectrodeposits," MEMS (2009) 96 (DOI: 10.1109/MEMSYS.2009.4805328).

[4] P. Malcovati, et al:: in Nyquist AD Converters, Sensor Interfaces, and Robustness, ed. P. Malcovati (Springer, New York, 2013) 149-174.

[5] J. Citakovic, et al.: "A compact CMOS MEMS microphone with $66 \mathrm{~dB}$ SNR," ISSCC Dig. Tech. Papers (2009) 350 (DOI: 10.1109/ISSCC.2009.4977452).

[6] J. F. Dickson: "On-chip high-voltage generation in MNOS integrated circuits using an improved voltage multiplier technique," IEEE J. Solid-State Circuits 11 (1976) 374 (DOI: 10.1109/JSSC.1976.1050739).

[7] J. T. Wu, et al.: "1.2 V CMOS switched-capacitor circuits," ISSCC Dig. Tech. Papers (1996) 388 (DOI: 10.1109/ISSCC.1996.488729).

[8] J. T. Wu and K. L. Chang: "MOS charge pumps for low-voltage operation," IEEE J. Solid-State Circuits 33 (1998) 592 (DOI: 10.1109/4.663564).

[9] J. Shin, et al:: "A new charge pump without degradation in threshold voltage due to body effect," IEEE J. Solid-State Circuits 35 (2000) 1227 (DOI: 10. 
1109/4.859515).

[10] D. S. Hong and M. N. El-Gamal: "Low operating voltage and short settling time CMOS charge pump for MEMS applications," ISCAS (2003) 281 (DOI: 10.1109/ISCAS.2003.1206254).

[11] A. H. Alameh, et al.: "A reconfigurable charge pump in $0.13 \mu \mathrm{m}$ CMOS for agile MEMS actuation,” ICECS (2014) 670 (DOI: 10.1109/ICECS.2014. 7050074).

[12] B. Razavi: Design of Analog CMOS Integrated Circuits (McGraw-Hill, New York, 2001) 706.

\section{Introduction}

A microelectromechanical systems (MEMS) microphone is an acoustic-to-electric transducer or sensor that converts an acoustic signal to an electrical signal. MEMS microphones are used in many applications, such as telephones, hearing aids, mobile phones, and personal audio systems [1]. An MEMS microphone, whose simplified structure is shown in Fig. 1a [2, 3], consists of 2 conductive plates. The bottom plate is fixed and immovable, while the top plate can vibrate with sound pressure, producing a capacitance variation. An acoustic signal is transformed into a capacitance variation.

The interface circuit for an MEMS microphone, whose block diagram is shown in Fig. 1b [4], typically consists of some sort of preamplifier followed by an analog-to-digital converter (ADC). A charge pump is usually required to generate the microphone bias voltage $V_{B}$.

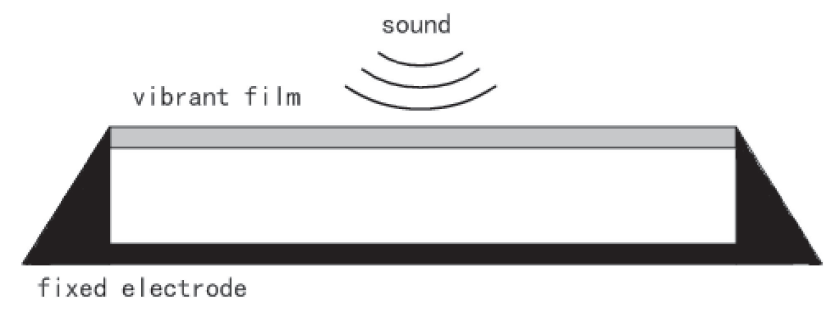

(a)

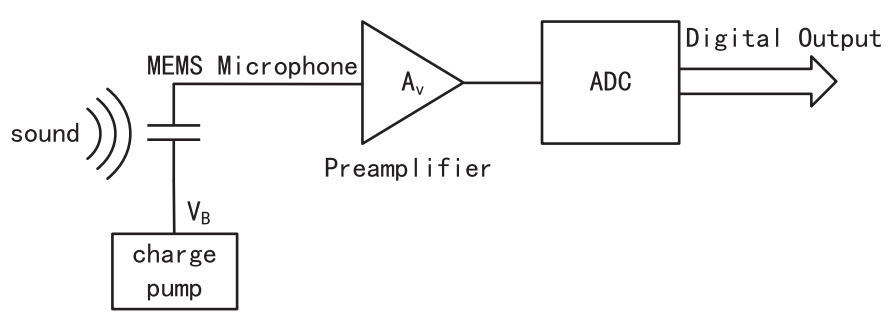

(b)

Fig. 1. (a) The basic structure of an MEMS capacitor; (b) The typical block diagram of interface circuits for an MEMS microphone.

To isolate an MEMS capacitor, a very large resistor $R_{b}$ (on the order of gigaohms) is connected between the microphone and charge pump [1]. If $R_{b}$ is infinite, the charge on an MEMS capacitor can be regarded as constant. Based on a constant-charge assumption, the output voltage $V_{\text {out }}$ can be expressed as 


$$
V_{\text {out }}=\frac{\Delta C \cdot V_{B}}{\left(C_{\text {mic }}+C_{p}-\Delta C\right)},
$$

where $\Delta C$ is the capacitance variation of the MEMS microphone, $V_{B}$ is the bias voltage, $C_{m i c}$ is the capacitance of the MEMS capacitor, and $C_{p}$ is the parasitic capacitance of the preamplifier and MEMS capacitor. Hence, the capacitance variation is transformed into an electrical signal.

As shown in eq. 1, the amplitude of $V_{\text {out }}$ depends on $V_{B}$. Therefore, to increase the microphone sensitivity, the value of $V_{B}$ must be high, typically ranging from $8 \mathrm{~V}$ to $12 \mathrm{~V}$ [5]. Consequently, a charge pump is usually required to generate the desired value of $V_{B}$.

In this paper, the supply voltage $V_{D D}$ is $1.8 \mathrm{~V}$. Some of the main parameters of the charge pump are shown in Table I.

Table I. Parameters of charge pump

\begin{tabular}{l|l|l}
\hline Parameter $($ Symbol $)$ & Value & Note \\
\hline Input voltage $\left(V_{\text {in }}\right)$ & $0.7 \sim 1.4 \mathrm{~V}$ & Limited by the supply voltage \\
\hline Output voltage $\left(V_{\text {out }}\right)$ & $8 \sim 12 \mathrm{~V}$ & $\begin{array}{l}\text { i) To improve the amplitude of the output voltage } \\
\text { ii) Considering the withstand voltage of MEMS }\end{array}$ \\
\hline Power consumption & $<100 \mu \mathrm{W}$ & To reduce power consumption \\
\hline
\end{tabular}

Section II will introduce the working principle of the Dickson charge pump, analyze its defects in detail, and briefly list some attempts to correct them. Sections III and IV will explain the principles of operation and the measurement results, respectively, of the fabricated charge pump. Finally, conclusions will be discussed in section $\mathrm{V}$.

\section{Analysis of existing charge pump topologies}

In 1976, Dickson invented charge pump circuits [6], as shown in Fig. 2. Since diode-connected MOS transistors are employed, the charges can be pushed only in 1 direction. The pair of pumping clocks $\Phi_{\mathrm{I}}$ and $\Phi_{\mathrm{II}}$ are non-overlapping and have an input voltage amplitude of $V_{i n}$. The clocks push the charge voltage upward through the transistors. Considering the impact of parasitic capacitance and the current loading, the voltage fluctuation at each pumping node $\Delta V$ can be expressed as

$$
\Delta V=V_{\text {in }} \frac{C}{C+C_{p}}-\frac{I_{o}}{f \cdot\left(C+C_{p}\right)},
$$

where $C$ is the capacitance of the coupling capacitor, $C_{p}$ is the parasitic capacitance associated with each pumping node, $f$ is the frequency of the pumping clocks, and $I_{O}$ is the output current loading.

When $\Phi_{\mathrm{I}}$ goes high and $\Phi_{\mathrm{II}}$ goes low, the voltage at node 1 settles to $V_{1}+\Delta V$, and the voltage at node 2 settles to $V_{2}$, where $V_{1}$ and $V_{2}$ are the steady-state lower voltages at nodes 1 and 2 . Both $\mathrm{MD}_{1}$ and $\mathrm{MD}_{3}$ are reverse-biased, and the charges are being pushed from node 1 to node 2 through $\mathrm{MD}_{2}$. 


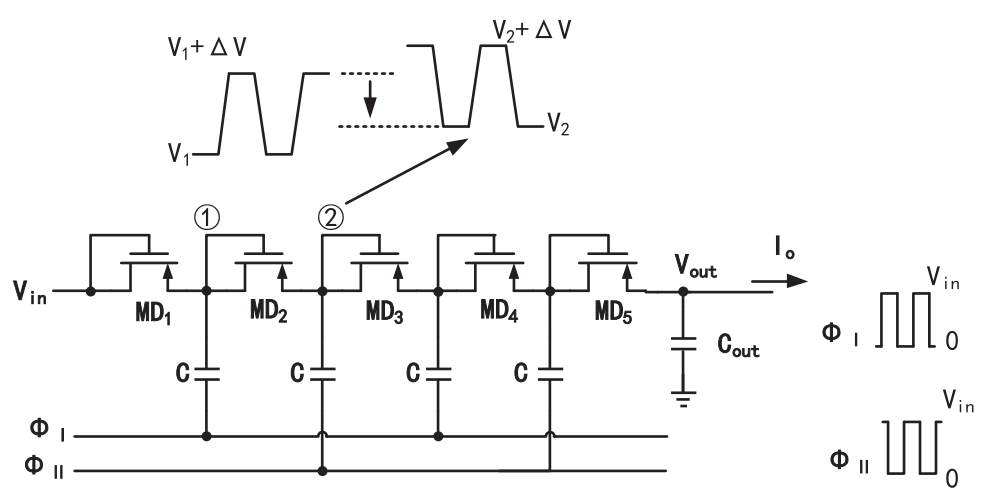

Fig. 2. Dickson charge pump circuits.

The necessary condition for the Dickson charge pump to function is that $\Delta V$ is larger than the threshold voltage of transistor $V_{t h}$, which can be expressed as

$$
\Delta V>V_{t h} .
$$

The voltage pumping gain for the second pumping stage $G_{V 2}$ is defined as the voltage difference between nodes 1 and 2, which can be expressed as

$$
G_{V 2}=V_{2}-V_{1}=\Delta V-V_{t h 2},
$$

where $V_{t h 2}$ is the threshold voltage of $\mathrm{MD}_{2}$, modified by the body effect. If $\Delta V$ is not much larger than $V_{t h}$, the body effect cannot be neglected, especially at the high-voltage nodes. In fact, the circuit's output voltage reaches its maximum when the body effect causes the threshold voltage to be equal to $\Delta V$. Therefore, the final output voltage is limited by the body effect, and the voltage pumping gain per stage, $G_{V}$, is reduced by $V_{t h}$.

In conclusion, the traditional Dickson charge pump circuit has 2 serious defects:

1) The voltage pumping gain per stage, $G_{V}$, is reduced by the threshold voltage $V_{t h}$.

2) The final output voltage $V_{\text {out }}$ is limited by the body effect.

To repair defect 1, Jieh-Tsorng Wu proposed a charge pump using static charge transfer switches (CTSs) in 1996 [7]. As shown in Fig. 3, $\mathrm{MS}_{1}-\mathrm{MS}_{4}$ are the CTSs. By using the already established high voltage to control the CTS of the previous stage, the upper voltage of the input is equal to the lower voltage, and defect 1 is repaired. However, when MD is reverse-biased, there is a large reverse leakage current, since the CTS's are always connecting.

To remove the reverse leakage current, Jieh-Tsorng $\mathrm{Wu}$ proposed another charge pump using dynamic CTSs in 1998 [8]. To repair defect 2, Jongshin Shin [9] and David S. Hong [10] proposed a charge pump with a dynamic body bias voltage in 2000 and 2003, respectively. By using auxiliary transistors to set the body bias voltage, the circuit maintains a constant threshold voltage, but defect 1 still exists. Abdul Hafiz Alameh proposed a reconfigurable voltage doubler [11], which improves drive capability and has a controllable output voltage, but needs a relatively larger chip area and consumes more power. None of the above attempts can repair both defects at the same time. 


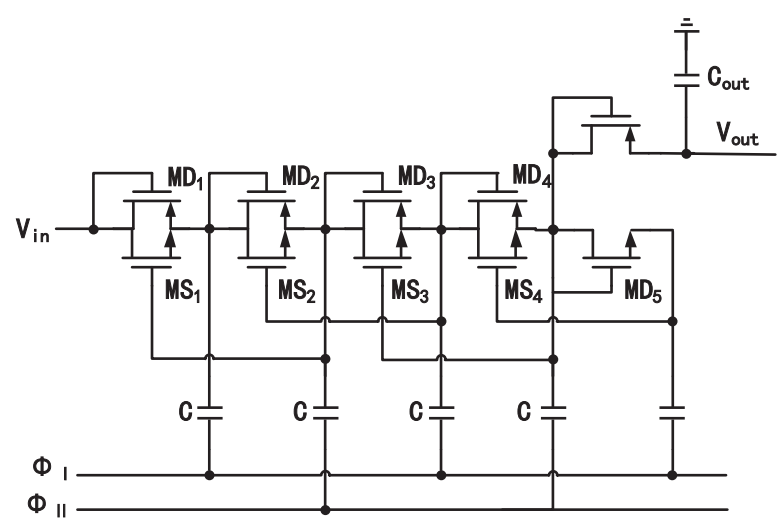

Fig. 3. Charge pump using static CTSs.

\section{Proposed charge pump circuits}

Fig. 4 is the block diagram of an energy-efficient charge pump. The pair of clocks, $c l k$ and $c l k \_n$, control each charge pump cell (CPC) and have a voltage amplitude equal to the supply voltage $V_{D D}$. The other pair of clocks, $c l k \_l v l$ and $c l k \_l v l \_n$, control each coupling capacitor and have a voltage amplitude equal to the input voltage $V_{i n}$. The main circuit consists of 12 CPCs and a special output stage. Every CPC can be seen as a switch, and $c l k$ and $c l k \_n$ causes them to be connected alternately.

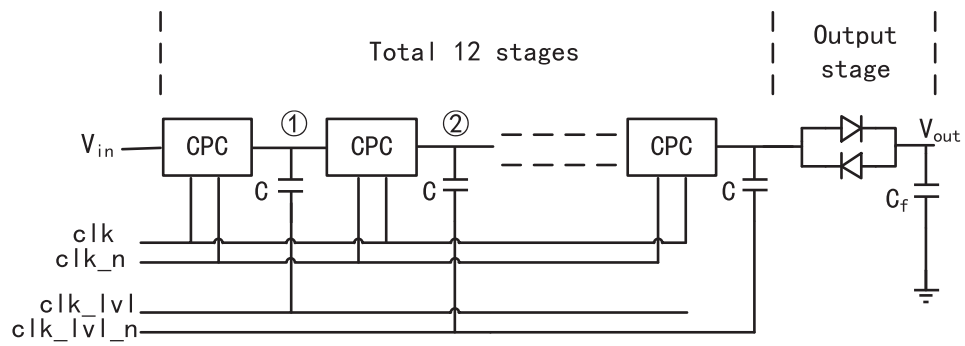

Fig. 4. Block diagram of an energy-efficient charge pump.

Fig. 5 shows the specific circuit of a CPC. MD is the charge transfer MOSFET. When $c l k$ goes low and $c l k \_n$ goes high, the gate voltage of MD drops, since the voltage drop across the capacitor cannot change suddenly, and MD connects. The charge is pushed from node 1 to node 2 through MD, and MD gets into deep liner region. The voltage of node 2 can be expressed as

$$
V_{2} \approx V_{1}+\Delta V
$$

where $V_{1}$ and $V_{2}$ are defined as the steady-state lower voltages at nodes 1 and 2, respectively. The charge on capacitor $C_{2}$ is pushed to capacitor $C_{1}$ through transistor $\mathrm{M}_{2}$ and $\mathrm{M}_{3}$. Then, the voltage of node $\mathrm{A} V_{A}$ can be expressed as

$$
V_{A}=V_{2}-\left|V_{t h 3}\right|,
$$

where $V_{t h 3}$ is the threshold voltage of $\mathrm{M}_{3}$.

When $c l k$ goes high and $c l k \_n$ goes low, $V_{A}$ rises by $V_{D D}$ while $V_{2}$ rises by $\Delta V$. 


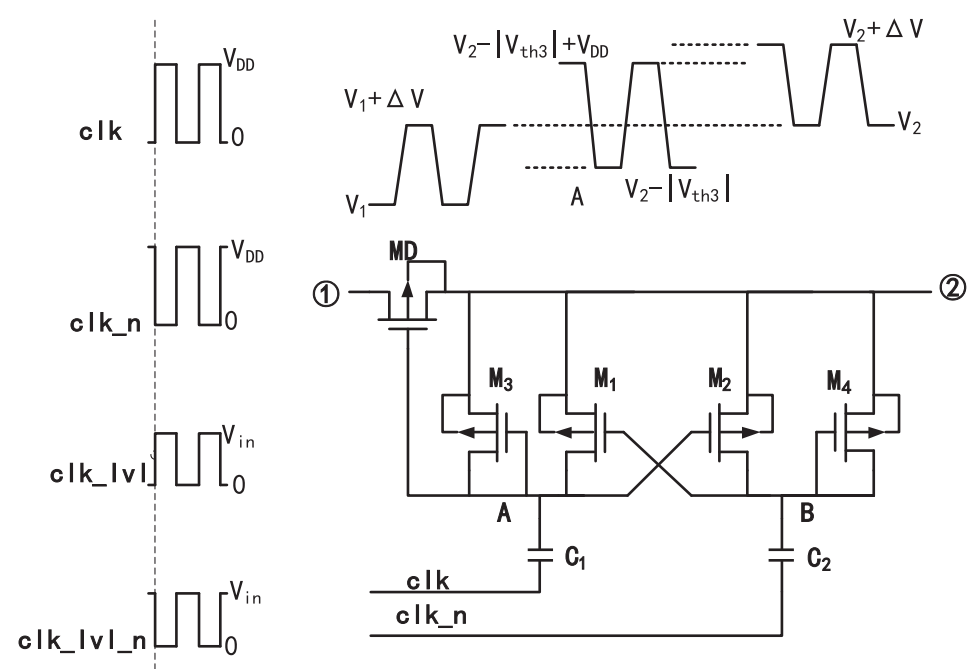

Fig. 5. Specific circuit of the CPC.

$$
V_{S G}=V_{2}-V_{A}=\left(V_{2}+\Delta V\right)-\left(V_{2}-\left|V_{t h 3}\right|+V_{D D}\right)=\Delta V-V_{D D}+\left|V_{t h 3}\right| .
$$

$V_{D D}$ is higher than $V_{i n}$, and $V_{i n}$ is higher than $\Delta V$, which can be expressed as

$$
V_{D D}>V_{\text {in }}>\Delta V \text {. }
$$

Ignoring the difference of the threshold voltage among transistors, $V_{S G}$ is lower than the absolute value of MD's threshold voltage $V_{\text {thd }}$. It can be expressed as

$$
V_{S G}<\left|V_{t h d}\right| .
$$

Since the MD cannot conduct and has no reverse leakage charge, the energyefficient charge pump eliminates defect 1 .

As shown in Fig. 5, the CPC employs positive channel metal-oxide semiconductor (PMOS) only and the transistors shares substrate. The voltage of the substrate, $\mathrm{N}$-well, is equal to $V_{2}$. Employing PMOS only can avoid latch-up. Since the source and substrate of MD are connected, the body effect on MD can be removed. This circuit also has the advantage that the charge can flow through drainsub parasitic PN junction and reduce the setup time.

The output stage is shown in Fig. 4. The proposed circuit employs 2 antiparallel diodes as the large resistor $R_{b}$. The I-V characteristic of the diode showes that charge pump circuits charge the MEMS capacitor rapidly when the voltage drop across the diode is large; while the voltage drop is low, the anti-parallel diodes can be equivalent to a larger resistor [12]. The anti-parallel diodes isolates the MEMS capacitor well at steady-state. In addition, there is a filter consisting of the equivalent resistor and MEMS capacitor. The charge accumulated on the MEMS microphone do not change by much because of the large RC time constant. It reduces the ripple at the output voltage. Furthermore, the output stage saves chip area and reduces the setup time.

This paper proposes an energy-efficient charge pump for an MEMS microphone. By employing an extra pair of clocks to make MD work as a switch, the proposed circuit repairs defect 1 . Furthermore, by sharing $\mathrm{N}$-well and connecting to the output voltage of the CPC, the proposed circuit repairs defect 2 . In addition, the output stage employs anti-parallel diodes, which can act as an equivalent resistor 
and reject the ripple of output voltage. The proposes circuit can cascade with many stages because there is no degradation in the threshold voltage due to body effects, and it generates higher output voltage.

\section{Measurement results}

The proposes circuit is fabricated in a $0.18 \mu \mathrm{m}$ CMOS process; the block diagram of the overall test circuit is shown in Fig. 6. The bandgap voltage circuit produces a reference voltage $V_{b g}$, and the external circuit provides the input signal for a digitalto-analog converter (DAC) through an inter-integrated circuit $\left(\mathrm{I}^{2} \mathrm{C}\right)$. The DAC generates an input voltage $V_{\text {in }}$ from $0.7 \mathrm{~V}$ to $1.4 \mathrm{~V}$. Fig. 7 shows a photograph of the energy-efficient charge pump; the chip area is $0.35 \mathrm{~mm} \times 0.125 \mathrm{~mm}$.

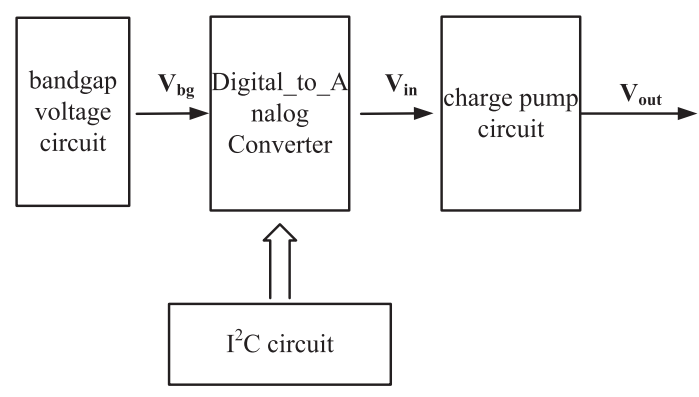

Fig. 6. Block diagram of overall test circuit.

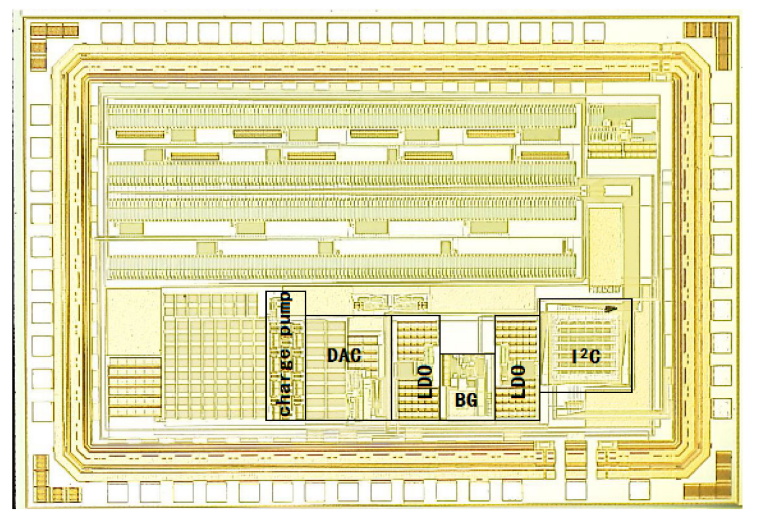

Fig. 7. Photograph of the energy-efficient charge pump.

Fig. 8 shows the measurement result of the proposed circuit. With $V_{\text {in }}$ ranging from $0.7 \mathrm{~V}$ to $1.4 \mathrm{~V}$, the output voltage ranges from $5.8 \mathrm{~V}$ to $12.5 \mathrm{~V}$. The output voltage has approximately $196 \mu \mathrm{V}$ of ripple, peak-to-peak, whereas the maximum power consumption is only $62.7 \mu \mathrm{W}$ with a $2-\mathrm{pF}$ load capacitor.

The operating clock is set at $2.4 \mathrm{MHz}$. The lower the working clock frequency, the longer the setup time, and the higher the working clock frequency, the greater the power consumption. As shown in Fig. 8(b), the setup time is $289 \mu$ s $(10 \%$ to 90\%), which meets the application scenarios. Without the discharge circuit, the setup time is much shorter at a second time and the power consumption can be reduced. 


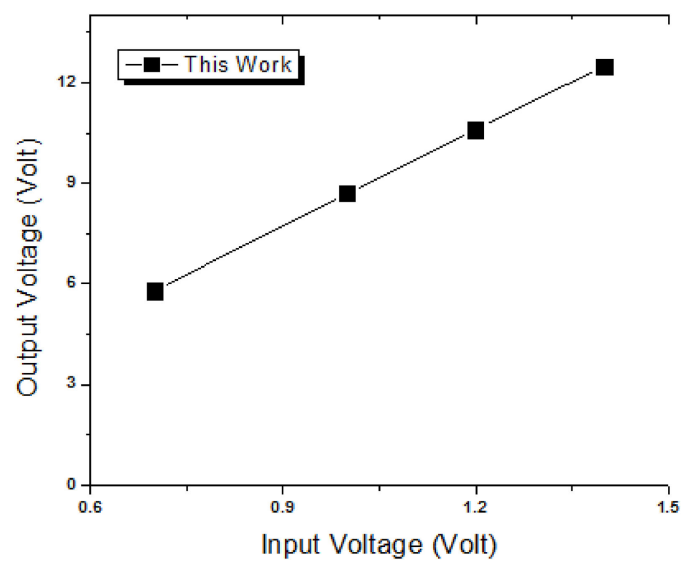

(a)

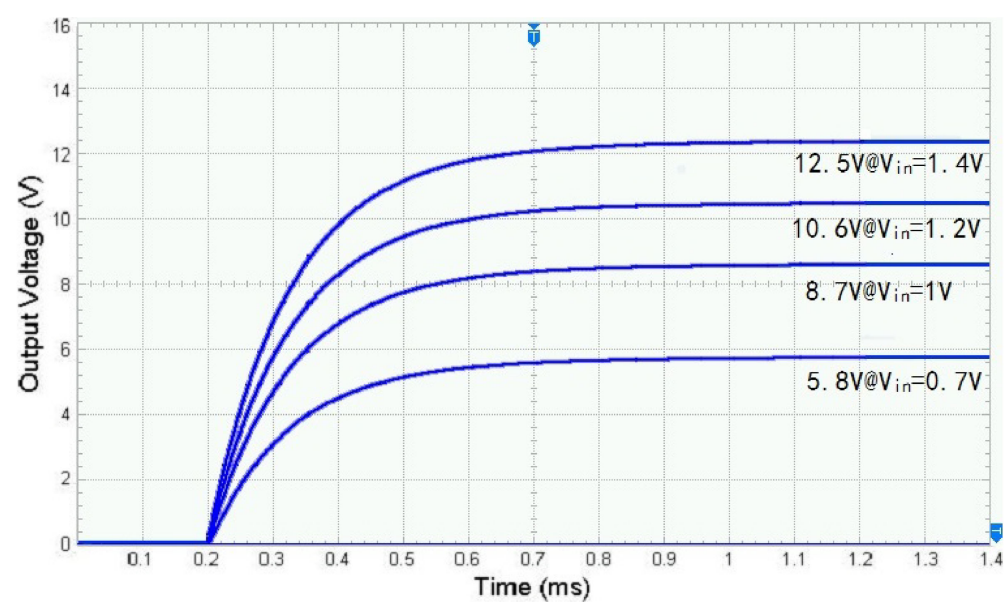

(b)

Fig. 8. (a) Measured output voltage with different input voltages; (b) measured output voltage versus setup time.

Table II summarizes the performance of the charge pump compared to those reported in other papers. As shown in Table II, the proposed circuit has a lower power consumption, lower ripple, and smaller chip area. The output voltage is roughly equal those reported in other papers. Ultimately, the proposed circuit is more suitable for an MEMS microphone.

Table II. Charge pump performance summary and comparison.

\begin{tabular}{l|l|l|l}
\hline Parameter (unit) & This work & {$[8]$} & {$[9]$} \\
\hline CMOS technology $(\mu \mathrm{m})$ & 0.18 & 0.18 & 0.13 \\
\hline Input Voltage $(\mathrm{V})$ & 1.4 & 1.2 & 1.2 \\
\hline Clock Frequency $(\mathrm{MHz})$ & 2.4 & 75 & 50 \\
\hline Number of Stages & 12 & 16 & 8 \\
\hline Max Output Voltage $(\mathrm{V})$ & 12.5 & 14.8 & 10.62 \\
\hline Ripple Voltage $(\mathrm{mV})$ & 0.196 & 150 & 48 \\
\hline Rise Time $(\mu \mathrm{s})$ & 289 & 65 & 1.56 \\
\hline Power Consumption $(\mu \mathrm{W})$ & 62.7 & 759 & 220 \\
\hline Area $(\mathrm{mm} \times \mathrm{mm})$ & $0.35 \times 0.125$ & $0.8 \times 0.9$ & $0.215 \times 0.3$ \\
\hline
\end{tabular}




\section{Conclusions}

The charge pump used for the MEMS microphone requires a high output voltage and low power consumption. This paper proposed a new charge pump that repairs the 2 main defects of traditional charge pump circuits. The proposed circuit is fabricated in a $0.18 \mu \mathrm{m}$ CMOS process. As seen in the results, the output voltage ranges from $5.8 \mathrm{~V}$ to $12.5 \mathrm{~V}$ with a $0.7 \mathrm{~V}$-to- $1.4 \mathrm{~V}$ input and a $2-\mathrm{pF}$ load capacitor, and the power consumption is only $62.7 \mu \mathrm{W}$. Compared to charge pump proposed in other papers, this charge pump has advantages in power consumption, ripple voltage, and chip area, and better suits the MEMS microphone.

\section{Acknowledgments}

This work is supported by the National Natural Science Foundation of China (No. 61404030). 